\title{
Improving the Reliability of Decision-Support Systems for Nuclear Emergency Management by Leveraging Software Design Diversity
}

\author{
Tudor B. Ionescu and Walter Scheuermann \\ Institut für Kernenergetik und Energiesysteme (IKE), Stuttgart, Germany
}

\begin{abstract}
This paper introduces a novel method of continuous verification of simulation software used in decision-support systems for nuclear emergency management (DSNE). The proposed approach builds on methods from the field of software reliability engineering, such as N-Version Programming, Recovery Blocks, and Consensus Recovery Blocks. We introduce a new acceptance test for dispersion simulation results and a new voting scheme based on taxonomies of simulation results rather than individual simulation results. The acceptance test and the voter are used in a new scheme, which extends the Consensus Recovery Block method by a database of result taxonomies to support machine-learning. This enables the system to learn how to distinguish correct from incorrect results, with respect to the implemented numerical schemes. Considering that decision-support systems for nuclear emergency management are used in a safety-critical application context, the methods introduced in this paper help improve the reliability of the system and the trustworthiness of the simulation results used by emergency managers in the decision making process. The effectiveness of the approach has been assessed using the atmospheric dispersion forecasts of two test versions of the widely used RODOS DSNE system.
\end{abstract}

ACM CCS (2012) Classification: Information systems $\longrightarrow$ Information systems applications $\rightarrow$ Decision support systems $\rightarrow$ Expert systems

Keywords: software reliability, decision-support, simulation, safety-critical, machine-learning

\section{Introduction}

An atmospheric dispersion simulation code (ADSC) is a computer program, which implements an atmospheric dispersion model (ADM).
In the aftermath of the Chernobyl nuclear accident in 1986, atmospheric dispersion models and simulation codes were used to assess the radiological situation in Western Europe, which was one of the worst affected areas. On this occasion, at least two important aspects concerning ADMs and ADSCs became evident.

Firstly, atmospheric dispersion models can be used for (1) assessing the radiological situation any time after the release of nuclear trace species, (2) supporting decision-makers in the process of deciding upon and implementing appropriate counter-measures, such as the distribution of iodine tables or the evacuation of severely affected areas, while the accident is unfolding, and (3) training for nuclear emergencies (i.e., drills). These systems produce forecasts of the atmospheric dispersion of radioactive materials using input data from various sources, including nuclear power-plants, weather forecast centers, and radiological measurement stations. Dispersion forecasts are used by government emergency response committees and nuclear power-plant operators in preparing for and managing nuclear emergencies.

Secondly, the forecasts of different ADMs, as well as the results of different ADSCs implementing even the same model, differ to a great extent qualitatively and quantitatively. In response to this situation, the International Atomic Energy Agency (IAEA) recommended the review and inter-calibration of the models and simulation codes for the short-ranged and longranged atmospheric transport of radioactive nu- 
clides. More recently, the European Commission also funded a project, called ENSEMBLE [1] with the aim of developing a methodology for assessing the reliability of several medium and long-range atmospheric dispersion models currently in use in the European Union and other countries of the world. The outcome of this work was a multi-model approach, called ensemble dispersion modeling (EDM). The system aggregates results produced by different ADSCs implementing various models, and outputs a unified result based on selecting the $p^{t h}$ percentile result from the ensemble for each time step and each location of the monitored area.

\subsection{Motivation}

Germany, as well as other countries, in case of a significant release of radioactive materials into the atmosphere, the decision on which countermeasures to take and which sectors of the monitored area to evacuate first is taken based on these forecasts. Thus, simulationbased decision-support software systems must be regarded as safety critical. Therefore, it is necessary to investigate the reliability of such systems from a software reliability point of view. Section 4.1.1.2 of the NASA-STD-8719. 13B standard for safety-critical software [2] provides a list of criteria for determining whether a software component or system must be considered safety critical. Accordingly, it suffices for the software component in question to meet just one of the listed criteria in order for it to be regarded as safety critical. Paragraph 4.1.1.2.a. states that as soon as the software "processes data or analyzes trends that lead directly to safety decisions", it must be considered safety critical. Moreover, note $4-1$ from the same standard specifies that "if data is used to make safety decisions (either by a human or the system), then the data is safety-critical, as is all the software that acquires, processes, and transmits the data". Decision support systems for managing nuclear emergencies (henceforth referred to as DSNE systems) are in use in all European countries producing nuclear energy - see for example [1] for a comprehensive list. They are safety critical because erroneous dispersion simulation results could lead to false assumptions about the dispersion of radioactive pollutants and may thus bias evacuation priorities.

\subsection{Proposed Approach}

Considering the simple fact that ensemble forecasting systems are a paradigmatic example of the software design diversity principle, the primary aim of this work is to assess the possibility of detecting latent software faults in the dispersion models participating in ensemble forecasting systems using a new software design diversity method. The current approach helps improve the reliability of ADSCs by allowing the effective automated continuous verification [3] of these simulation codes. The proposed approached is based on combining the N-Version Programming (NVP) [4] and Recovery Blocks (RB) [5] methods while developing a completely new voting scheme as well as a new acceptance test for dispersion simulation results.

The remainder of the paper is organized as follows. We first briefly review the ADMs implemented in the RODOS system [6] and then provide a literature review on verification methods for atmospheric dispersion simulation codes. We then review the current debate concerning software design diversity methods and argue that they are well suited for the case of dispersion forecasting systems. We introduce a new acceptance test and taxonomy-based voter, which enable the application of the NVP and $\mathrm{RB}$ methods for the continuous verification of dispersion simulation codes. Finally, we evaluate the approach using two ensembles of artificially generated data and discuss the results of the evaluation.

\section{Materials and Methods}

\subsection{Atmospheric Dispersion Modeling}

The atmospheric dispersion of trace species is governed by meteorological events. Wind and turbulence are the most influential forces in the dispersion process. In the following, three wellestablished ADMs will be briefly reviewed. All these models share the property that they represent analytical or numerical solutions to the diffusion-advection (also called transport) equation [7] - the most common physical model used to simulate the atmospheric dispersion of 
trace species. This property is an essential prerequisite for the applicability of the software design diversity methods proposed in this work to the simulation codes implementing any of the models considered here.

\subsubsection{The Gaussian Plume Model}

The Gaussian Plume Model (GPM) is the oldest dispersion model with practical applicability. The model is based on an analytical solution to the transport equation, which accounts for the airborne transport of trace species. This transport occurs through the mean air flow (i.e., wind), in which case it is called advection, and through turbulent movement. Turbulence can disperse trace species in all three directions of space represented by the three axes of the Cartesian system. This process is called diffusion. Any dispersion of substances which does not take place along the main flow is referred to as turbulent diffusion. The substances contained within an air volume are quantified by their concentration per unit volume, $c\left[\mathrm{~kg} \mathrm{~m}^{-3}\right]$.

\subsubsection{The Lagrangian Particle Model}

The Lagrangian particle dispersion model (LPDM) [8] was adopted by the scientific community and regulatory authorities by the end of the 90 s as the field's de facto standard [9]. The DIPCOT [10] simulation code, which is used in an operational DSNE system, represents an implementation of the Lagrangian particle model. In this model, an ensemble of particles is used to represent a much higher number of molecules of substance per particle released into the atmosphere (i.e., trace species). The particles also reflect the physical properties of the trace species they stand for. The monitored area is divided into a 3-dimensional regular grid for which a wind field is computed at the beginning of each time step. Then, according to the wind velocity and direction, within one time step each particle can move from one grid cell to another whereby its location is updated. In addition to the change of position, the particle will suffer a change in its mass due to gravity (dry deposition) and/or precipitation (wet deposition). Through dry and wet deposition the air concentration of the trace specie(s) represented by some particle will decrease according to first order differential processes.

\subsubsection{Puff Models}

Puff dispersion models are more accurate than Gaussian-Plume models and less time-consuming than the Lagrangian particle model. These models simulate the dispersion of trace species using a series of Gaussian puffs of different sizes. A puff contains a quantity of pollutant (characterized by its concentration) which is subject to turbulent diffusion and advection by a wind field in a way that is similar to how particles are transported. RIMPUFF [11] and ATSTEP $[12]$ are the two codes implementing puff models used to evaluate the current approach.

\subsection{The Verification and Validation of Atmospheric Dispersion Simulation Codes}

Much like any other software product, dispersion simulation codes must undergo a thorough verification and validation phase prior to being integrated into production systems. [13] provide an extensive overview of the best practices and methods for the verification and validation of simulation codes from the fields of computational engineering and physics. According to [13], code to code comparisons are only accepted as verification procedures provided that traditional and well accepted verification and validation procedures have been undertaken as well. An equivalent method to code to code comparisons has been used for comparing commercial seismic data processing software. In an extensive $\mathrm{N}$-version programming study (called the T-experiments) [14], the authors show that the different versions of the scientific software participating to the study compared led to overwhelming disagreement between results due to software problems.

The VDI guideline number 3945 [9] for environmental meteorology and atmospheric dispersion models proposes a comprehensive list of verification tests for codes implementing the Lagrangian particle model described in the guideline. These tests are aimed at verifying "whether the algorithm has been correctly implemented in a computer program (verification)". All tests are to be repeated 101 times with a different sequence of random numbers. One drawback of this set of verification tests is that it targets 
particle models only. That is, most of the tests cannot be applied to Puff models. Also, these tests can only be performed during the test and integration phase of the system because they require extensive preparation, many repetitions, and qualitative assessment of the results. Moreover, none of the tests can be easily automated. This makes them unsuitable for detecting errors in arbitrary dispersion simulation results produced by operational DSNE systems. In practice, the de facto standard method of verification is that of plausibility checks through visual inspection.

In [15] the authors propose simple processes and tests for improving the reliability and usefulness of models, while in [16] which also provides a good review of other relevant work targeting the software engineering practices within the EMS community, the authors recommend a comprehensive 10-step development and evaluation process for environmental models. Scientific software often has a lifetime of several decades and therefore the evolution of scientific software needs to be studied more thoroughly [17]. Different studies have pointed out that a quality-oriented software development culture may be considered less important in research institutes than model developments and refinements, considering that researchers are generally more interested in producing and publishing state-of-the-art research results than highquality software [14,18].

In [19] the authors define verification as "the determination that the code solves the chosen model correctly" and validation as "the determination that the model itself captures the essential physical phenomena with adequate fidelity". The same authors further argue that verification must always precede validation since otherwise any agreement between its results and experimental data is likely to be a product of chance.

In the field of atmospheric dispersion modeling, validation generally prevails over verification in most published studies. Perhaps, this is because most codes are old and verification is usually done only once in the early testing phase. Benchmarking (i.e., comparing the output of the code being verified to the outputs of wellestablished codes) is the most common verification method used in published work. Different experimental data sets are also available from the HARMO website ${ }^{1}$ along with validation and verification guidelines. Within the atmospheric dispersion modeling community there is a strong credo that verification must always imply comparisons with measurement data. In this context, considering that in software testing the aim is to also cover possibly unlikely input cases to the end of finding software faults, as for example in random testing [20], a paradox becomes evident: while measurement data from experiments and accidents, such as the ones from Chernobyl and Fukushima, provide a gold standard for the evaluation of dispersion models, these datasets are limited in number. From a software testing perspective, this also limits the number and variety of input test cases considered for verification purposes. This practice contrasts with the software testing principles based on test case coverage criteria [21]. Test coverage criteria aim at stressing the limits of the software being tested to the end of finding more faults rather than making the software behave as expected for a limited set of input cases.

In [22] and [17] the authors point out that there are very few published methods and studies aimed at finding and removing pure code faults (or mistakes) from scientific software. They propose a new type of testing activity, called code scrutinization, which is to be carried out before verification and validation. The authors show that random and mutation testing can successfully be used for code scrutinization. This white-box testing method is well suited for the developers of stand-alone dispersion simulation codes. However, for DSNE systems, which incorporate several functionally redundant dispersion simulation codes developed by different institutes, the source code may not be available. Therefore, in the case of DSNE systems, most of the time only gray and black-box testing can effectively be carried out. The results of these testing activities are communicated to the developers of the respective codes in the form of the input cases that triggered disagreement between the codes implemented in the DSNE system.

\footnotetext{
${ }^{1}$ Initiative on "Harmonisation within Atmospheric Dispersion Modelling for Regulatory Purposes": http://www.harmo.org
} 


\subsubsection{Validation and Benchmarking Studies with the RODOS System}

The RODOS system encompasses three dispersion codes-ATSTEP, DIPCOT, and RIMPUFFdeveloped at different research institutes around the world. RODOS is used in several European countries (including Germany) as the official decision support system for nuclear disaster management. All three dispersion simulation codes used in RODOS have been subjected to validation using measurement data and to comparisons with other systems $[23,24,25,26]$. In the most recent validation study targeting the codes used in the RODOS system based on inter-code comparisons and comparisons with experimental data the author states that "the models should deliver similar results under [the considered] circumstances," while some results proved to be definitely wrong and some cases revealed significant discrepancies in the results of different models [27]. RODOS has also been evaluated using data from the Fukushima accident and compared to other similar systems on the basis of the Fukushima case [28,29]. One study also discusses the modifications that needed to be made to the RODOS system in order to support several release phases spread over several weeks, as was the case of the Fukushima accident [30]. This type of release had not been foreseen by the creators of the system before the Fukushima accident.

After the Fukushima accident, a large benchmarking study which comprised 9 atmospheric dispersion models, including the three RODOS models, used in Germany and Switzerland has been carried out [31]. In this study, 8 artificially generated input cases starting from simple meteorological conditions to realistic weather situations were formulated to define the boundary conditions for the calculations. This experiment is noteworthy not only because it uses many atmospheric dispersion codes, but also because 7 of the 8 input cases are artificially generated. Only one case, deemed more realistic by the authors, uses weather data predicted by the COSMO model of the German Weather Service.

All these studies show that, while RODOS is a well-regarded validated DSNE system, there are improvements to be made whenever a new data set becomes available or new codes are used in the comparison. This underpins the idea that continuous verification and better test coverage, which also includes unlikely input cases, can only improve the system over time.

\subsection{Multi-Model Ensemble Forecasting Systems}

Multi-model ensemble dispersion forecasting systems represent the state of the art approach in the ensemble dispersion forecasting community. In this approach, several functionally redundant simulation codes are used to produce an aggregated result, which is in fact selected using a voting scheme. While there is consensus in the community that ensemble systems produce better results than single version systems [32], there is a lively debate in literature about how to identify the models, which have a bad influence on the other members of the ensemble to the end of using a more reliable reduced ensemble of models. One strategy is to eliminate those results from aggregation which are deemed redundant with respect to their bias [33]. This strategy contrasts with the software design diversity principle discussed in the next section of this paper. Current best practices in multi-model dispersion forecasting ensembles suggest that model diversity and accuracy are key to providing more realistic and trustworthy results [34]. Here diversity is believed to be beneficial within a reduced ensemble when it produces a better result with respect to the gold standard (i.e., measured data). However good diversity relies on the accuracy of individual models, which must be assessed beforehand. The reduced ensemble approach is complementary to the weighting approach, where the results of all ensemble members are weighted prior to being used in computing an aggregated result [35].

\subsection{Software Design Diversity}

The Software design diversity principle is based on developing dissimilar functionally redundant software versions starting from the same basic set of requirements. It is conjectured that the more diverse the solutions, the lower will be the likelihood of common-cause failures. However, until now it was not possible to prove that failure-independence in functionally equivalent software components can be assured through the practice of independent development $[36,37]$. It 
has been argued that failure correlation strongly depends on the application of software engineering methods and best practices during the software development process, hence, the possibility of reducing the probability for failure correlation by improving the software development processes [38]. In [39] the authors proposed a model for why different developer teams tend to make the same mistakes based on the notion of "variation of difficulty" over the demand space. This model states that greater variation of difficulty increases the failure dependence between functionally redundant software versions. Another approach is to force diversity into the development process of the different versions - an approach which has been used for the Airbus A320 flight control software [40].

Currently, software design diversity methods are generally considered to improve the overall reality of the system, albeit at a cost that cannot be easily determined a priori [41]. The ongoing debate concerning the effectiveness of software design diversity is focused on the costs it involves over the costs of developing a reliable single version system [42]. In the case of atmospheric dispersion forecasting software, the cost issue is not at stake because dozens of independently developed simulation codes already exist and have been integrated in ENSEMBLE systems. Here, the problem lies in the way that simulation results are being compared.

Once several functionally redundant versions, of a program are available, it is possible to develop a new software design diversity method based on existing patterns from the domain of software reliability engineering, such as Recovery Blocks (RB) [5] or $\mathrm{N}$-version programming (NVP) [43]. Such methods require an acceptance test and a voting scheme, both of which will be introduced in the following sections.

\subsection{A New Acceptance Test for Atmospheric Dispersion Simulation Results}

The acceptance test proposed in this paper can be regarded as both an accounting check and a reasonableness test - see [44] for a classification of acceptance tests. The acceptance test checks that the distribution of the frequency function of all concentration or dose values obtained by dropping the spatial information from the dataset (i.e., by converting 2 and 3-dimensional matrices into one dimensional arrays of values) obeys a certain hypothetical distribution. The frequency function is obtained by generating a histogram of the values for all cells of the discretized monitor area. Using simple input cases for which the activation of software faults is highly unlikely, we found that the hypothetical frequency function has the shape of the Weibull distribution [45], as also noted in [46]. Intuitively, this means that (1) in any dispersion simulation result, the number of high concentration values must be much smaller than the number of low concentration values and (2) that the transition from one concentration level to another must be smooth and gradual. This represents the hypothesis of the proposed acceptance test, which was implemented in $\mathrm{R}^{2}$. After reducing the two-dimensional matrix containing the concentration or dose values to a onedimensional array (i.e., dropping the spatial information from the dataset) a goodness of fit test can be used with the resulting data set. The Kolmogorov-Smirnov goodness of fit test [47] proved to be suitable for an automated acceptance test for several reasons. The KolmogorovSmirnov (KS) test is a distribution-free goodness of fit test, which tests whether or not an empirical cumulative distribution function $(\mathrm{CDF})$ fits a hypothetical (expected) CDF. Compared to other statistics, the KS test is more intuitive and easier to implement. Simplicity is a desirable property of an acceptance test in a software reliability engineering sense. It also uses the empirical and hypothetical CDFs functions directly rather than contingency tables. Also, the hypothetical distribution of the concentration and dose values was unknown to us when we started the tests and, in theory, the KS test works with any hypothetical distribution. Finally, not as much emphasis was put on the selection of a particular statistical test as on the pragmatic implementation considerations. Any goodness of fit test that works with this kind of data and requires a simple implementation can be used.

Since the Weibull distribution is L-shaped it makes the application of an automated goodness of fit test difficult (in an L-shaped histogram the vast majority of the measurements fall into the first interval from the left). To overcome this

\footnotetext{
${ }^{2}$ Implementation of the acceptance test: https://clustio.googlecode.com/files/KS_Taxonomy_R_Scripts.pdf
} 
inconvenience, Weibull-distributed data samples can be transformed into exponentially distributed samples before applying the goodness of fit test. The transformation of the data set prior to applying a goodness of fit test is very common in the statistical practice [48].

\subsection{A New Taxonomy-Based Voter for Atmospheric Dispersion Simulation Results}

One way of obtaining taxonomies from arbitrary data is through hierarchical clustering [49]. Hierarchical clustering has an important advantage over other clustering methods (such as partitional clustering); namely, it allows for exact comparisons between resulting taxonomies. By relating new results to existing ones, a taxonomy of intra-model results can be obtained. By intra-model results it is meant that all results participating to the test are produced by the same dispersion simulation code. The taxonomy represents the memory (or history) of the dispersion code whereas the process of classifying a new result into an existing taxonomy may be regarded as a learning process. The most important thing here is to be certain that new results which are to be incorporated into a taxonomy (i.e., what is being learned) are not flawed; for, once accepted in the taxonomy, they are taken to be correct.

\subsubsection{A New Metric for Atmospheric Dispersion Simulation Results}

A metric is a function $d$ defining a distance between arbitrary individuals from a population. A data set with an associated metric is called a metric space and is denoted by $(M, d)$. Dispersion simulation results are provided in form of two or three-dimensional matrices whereby each matrix element corresponds to one area or volume element of the regular grid used to discretize the monitored area. The values contained in these matrices can be provided in arbitrary units. In the case of radioactive trace species, the values corresponding to each grid cell can be one of integrated activities/doses or as activity/dose rates expressed in either [Bq] (Bequerel) / [Sv] (Sievert) or $[\mathrm{Bq}] /[\mathrm{Sv}]$ per unit of time.

Dispersion plumes can be regarded as distributions of continuous variables in a finite space delimited by the monitoring area. In this case the residual sum of squares (RSS) can be used as a metric on the result space by letting one of the plumes account for the observed values $y_{i}$ and the other one for the estimated values $f\left(x_{i}\right)$ :

$$
R S S=\sum_{i=1}^{n}\left[y_{i}-f\left(x_{i}\right)\right]^{2} .
$$

The normalized version of the RSS metric can be used with the results of arbitrary dispersion codes, since all of them produce matrix-based outputs. The use of a normalized metric also guarantees that the distances between arbitrary result spaces will be preserved (i.e., isometry).

Since dispersion simulations are iterative, after each time step a new result matrix becomes available. When comparing intra-model results, one can choose to include any number of time steps in the calculation of the normalized RSS metric. In statistics it is common practice to perform transformations on the data prior to analyzing them. In the case of dispersion simulation results, applying a power transformation with a power $0<p<1$ will smoothen and level the data since very large values will become smaller and sub-unitary values will increase. Given a power $0<p<1$ and two dispersion simulation results $r_{1}$ and $r_{2}$ defined on an $N$ by $M$ cell monitored area for a number of $T S$ time steps, the generalized RSS distance is given by:

$$
\operatorname{GRSS}\left(r_{1}, r_{2}\right)=\sqrt{\sum_{t=1}^{i \leq T S} \sum_{i=1}^{i \leq N} \sum_{j=1}^{j \leq M}\left(r_{1}[t, i, j]^{p}-r_{2}[t, i, j]^{p}\right)^{2}}
$$

The advantage of this metric over other metrics is that it provides a high level of flexibility by letting the practitioner select the most suitable value of $p$.

\subsubsection{The Reference Input Case Ensemble}

The reference input case ensemble will serve as the basis for the evaluation of the methods introduced in this work. The main requirements to the reference input case ensemble are the following:

- It shall cover the entire monitored area surrounding the chosen point of emission;

- Input cases shall be defined such that from one case to another all of the following input parameters are varied: wind direction 
(WD), wind speed (WS), accident category (AC), and diffusion category (DC);

- The variation shall be equidistant (where applicable);

- The wind direction shall be varied such that neighboring plumes overlap to some extent;

- The number of simulation steps and the simulated time shall not be varied from one input case to another.

The "each-used" test case coverage criterion [21] yielded the list of input cases shown in Figure 1. The names of the cases are coded as follows: the first letter represents the turbulent diffusion category (A to F), the next three digits represent the wind direction, the number following the letter ' $\mathrm{S}$ ' stands for the wind speed in $\mathrm{m} / \mathrm{s}$, and the number following the letter ' $\mathrm{F}$ ' represents the release (or accident) category according to the DSRA risk study [50]. Table 1 contains information about the amount and type of radioactive substance that is released into the atmosphere depending on the release category. The categories are ordered with respect to the total activity of the released radionuclides. In consideration of the previously stated requirements to the reference input case ensemble, the point of emission was chosen to be the KKP2 (Philippsburg 2) reactor in Germany and the radius of the monitored area $16 \mathrm{~km}$. The area surrounding the Philippsburg 2 reactor is rather flat, so the topography of the monitoring area does not have great influence. The simulation duration was chosen to be 2 hours divided into 12 time steps of 10 minutes each. The time window of 2 hours was chosen in accordance with

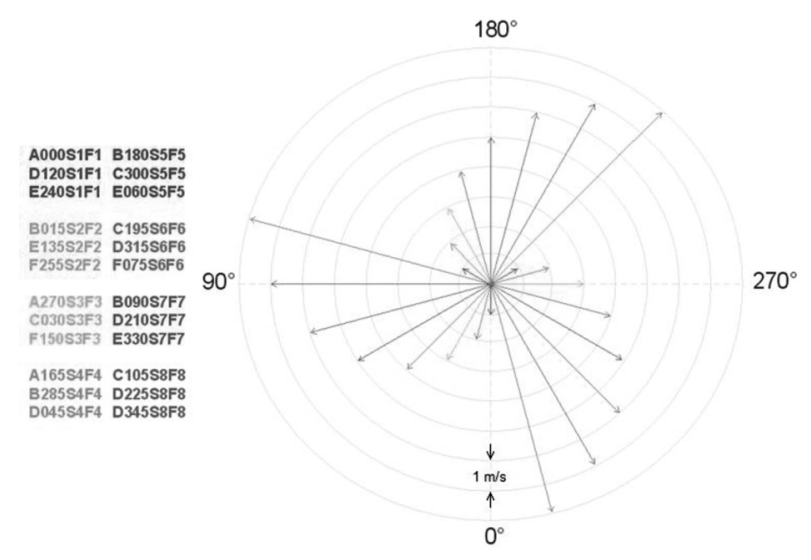

Figure 1. Sketch of the reference input ensemble.

the modeling area of $16 \mathrm{~km}$ in radius, which is also within the scale of the Fukushima evacuation area. At the highest considered wind speed of $8 \mathrm{~m} / \mathrm{s}(28.8 \mathrm{~km} / \mathrm{h})$, the trace species emitted at $t=0$ would reach the boundary of the modeling area in less than 30 minutes. This is sufficient to activate software faults related to erroneously posed boundary conditions, which may occur after part of the trace species exit the monitoring area. Ill-posed boundary conditions can lead to a violation of the mass conservation principle. This principle is fundamental in the numerical simulation of partial differential equations, such as the transport equation, and thus provides a suitable verification criterion. The data used in the reference input case ensemble are generated using the meteorological preprocessor of the RODOS system and are thus artificial. It is a common practice in running "what if" simulation scenarios for users of

Table 1. The eight release categories and corresponding amounts of radioactive materials with respect to the total amount present in the reactor according to the DSRA risk study [50]. A star marks the release categories corresponding to core meltdown accidents.

\begin{tabular}{|c|c|c|c|c|c|c|c|c|c|}
\hline $\begin{array}{c}\text { Release } \\
\text { category }\end{array}$ & $\begin{array}{c}\text { Emission } \\
\text { height } \\
{[\mathbf{m}]}\end{array}$ & $\begin{array}{c}\text { Duration } \\
{[\mathbf{h}]}\end{array}$ & $\begin{array}{c}\text { Noble } \\
\text { gas [\%] }]\end{array}$ & $\begin{array}{c}\text { Iodine } \\
{[\%]}\end{array}$ & $\begin{array}{c}\text { Alkali } \\
\text { metals } \\
{[\%]}\end{array}$ & $\begin{array}{c}\text { Tellurium } \\
\text { Antimony } \\
{[\%]}\end{array}$ & $\begin{array}{c}\text { Alkaline } \\
\text { earth } \\
\text { metal [\%] }\end{array}$ & $\begin{array}{c}\text { Ruthenium } \\
\text { group } \\
{[\%]}\end{array}$ & $\begin{array}{c}\text { Lanthanides } \\
{[\%]}\end{array}$ \\
\hline \hline F8 & 100 & 6 & 0.02 & 0.00 & 0.00 & 0.00 & 0.00 & 0.00 & 0.00 \\
F6* & $100 / 10$ & 3 & 2.30 & 0.00 & 0.00 & 0.00 & 0.00 & 0.00 & 0.00 \\
F5* & 10 & 3 & 2.30 & 0.11 & 0.07 & 0.07 & 0.01 & 0.01 & 0.01 \\
F7 & 10 & 1 & 1.70 & 0.53 & 1.30 & 0.00 & 0.00 & 0.00 & 0.00 \\
F4* & 10 & 3 & 66.80 & 1.47 & 0.34 & 0.33 & 0.04 & 0.33 & 0.00 \\
F3* & 10 & 3 & 66.80 & 4.68 & 2.93 & 2.67 & 0.33 & 0.22 & 0.03 \\
F2 $^{*}$ & 10 & 3 & 66.80 & 27.12 & 19.32 & 12.68 & 2.31 & 1.13 & 0.17 \\
F1 $^{*}$ & 10 & 1 & 100 & 79.60 & 50.00 & 35.00 & 6.70 & 38.00 & 0.26 \\
\hline
\end{tabular}


the system to manually input the wind speed and direction, as well as the precipitation rate and the turbulence parameters.

The released activity which is given by the release category is taken into account when computing the GRSS metric for two arbitrary dispersion simulation results. The $p$ parameter of the GRSS metric allows one to control the influence (or weight) of the amount of released activity (i.e., concentration of substance) upon the reference taxonomy of results. For $0.4>p>1$, the weight of the activity is greater than that of the wind direction. It is therefore justified to vary the release categories, and thus the amount of released activity among the input cases from the reference ensemble.

\subsubsection{The Reference Taxonomy of Results}

Considering a set of $N$ intra-model dispersion simulation results (henceforth called ensemble of results), a distance matrix $D$ is obtained by computing all possible distances $d_{i j}$ between all result pairs $i j$. The distances are computed using the GRSS metric. Starting from the distance matrix $D$, a multitude of clustering methods can be used to build a taxonomic scheme of dispersion simulation results.

Figure 2 shows the surface plot of the 24 by 24 distance matrix corresponding to RIMPUFF results for the ensemble of 24 input cases introduced in the previous section as well as the taxonomy of results inferred by means of the centroid method. The results represent the time-integrated near-ground gamma submersion dose. The distances have been computed using the GRSS metric (for $p=1$ ) for all 12 time steps accounting for a 2 -hour simulation. The results taxonomy is represented as a phylogram. The lengths of the branches in the left hand side phylogram reflect the distances between the elements being clustered as provided in the distance matrix. By contrast, the branch lengths of the phylogram on the righthand side do not reflect the original distances from the matrix. Instead, this one's purpose is to show the topology of the taxonomic tree.

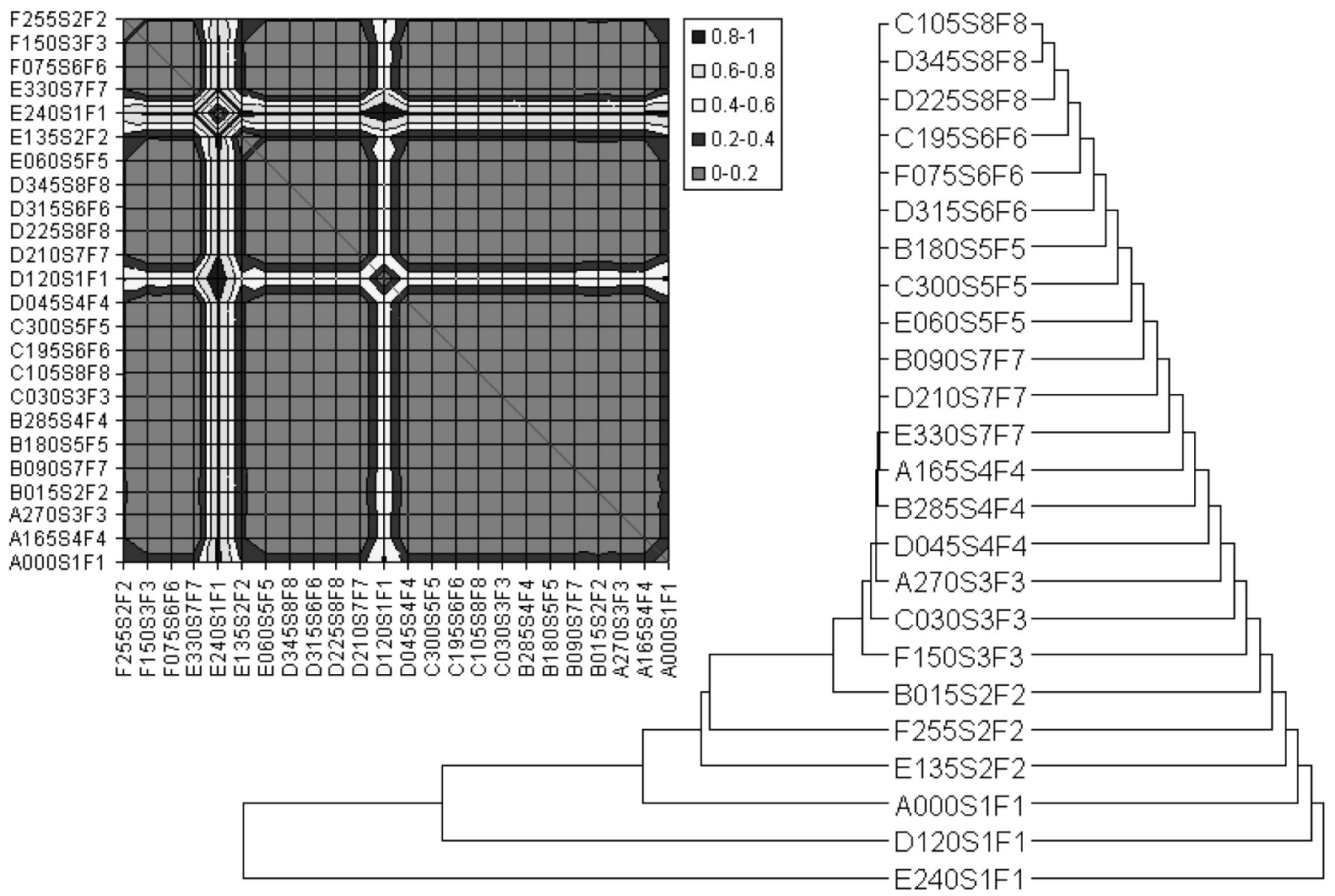

Figure 2. Surface plot of a $24 \times 24$ distance matrix computed from RIMPUFF results for the 24 input case reference ensemble, as well as the corresponding taxonomic tree built by means of the centroid method. The taxonomy is drawn as a phylogram with (left) and without (right) consideration of edge lengths. 
The phylogram has the topology of a caterpillar tree which reflects the order relations within the sample. The results are first ordered by the amount of the released radioactive materials specified by the release category (denoted "F1" to "F8" in the names of the input cases). The second ordering criterion appears to be the atmospheric stability class.

\subsubsection{Comparing Taxonomies of Results}

Dispersion simulation codes fulfill the two basic requirements of the $\mathrm{N}$-version programming paradigm: (1) they are developed and maintained by completely independent developer teams, and (2) they implement dispersion models which all approximate the solution to the transport equation. Hence, they start from the same basic functional requirement - solving the transport equation numerically. However, due to the inherent differences in the numerical schemes used for solving the transport equation, the results of the various dispersion codes will differ to such an extent that finding suitable thresholds and tolerance values to be used by voters can be rather difficult.

The aim of the current approach is to assess the degree of similarity (or dissimilarity) between the results of two or more arbitrary dispersion codes $C_{1} \ldots C_{n}$ by first constructing a taxonomy of results for each of them starting from the same ensemble of input cases $P=\left\{p_{1}, \ldots, p_{n}\right\}$ and then by comparing the resulting taxonomies rather than individual results. Using $P$ as the input case ensemble, each dispersion code $C_{k}$ will produce an ensemble of results $R\left(C_{k}\right)=$ $\left\{r_{1}\left(C_{k}\right), \ldots, r_{n}\left(C_{k}\right)\right\}$, whereby $p_{i}$ corresponds to $r_{i}\left(C_{k}\right)$ for $i=1, \ldots, n$. The next step is to build taxonomic trees for each result ensemble.

In order to build a taxonomy $T\left\{R\left(C_{k}\right)\right\}$ from the members of some result ensemble $R\left(C_{k}\right)$, the pair-wise distances between all of its members need to be computed using the metric of choice $d$ which gives a distance (or dissimilarity) matrix $D\left(C_{k}\right)=\left[d_{i, j}\right]$ for $i, j=1, \ldots, n$, as described in the previous section. In this context, what enables the implementing voters is the following conjecture:

If $C_{1}$ and $C_{2}$ are two dispersion simulation codes correctly implementing arbitrary numerical schemes for solving the transport equation, then there exists a distance metric $d_{i, j}$ defined on the metric space $(M, d)$ of the dispersion simulation results produced by $C_{1}$ and $C_{2}$ such that $T\left\{R\left(C_{1}\right)\right\} \equiv T\left\{R\left(C_{2}\right)\right\}$.

A dispersion code is considered to correctly implement a numerical scheme for solving the transport equation if and only if it conserves its mathematical properties (i.e., determinism, strict monotonicity, and continuity). Failing to do so is taken to be an indication of the presence of residual software faults in the code caused by the misinterpretation of the model or some other programming or computing error. The same principle applies to post-processing steps, such as the gamma submersion and effective dose calculation. Assessing the similarity between taxonomic trees requires a metric defined on the tree space. The Robinson-Foulds (RF) symmetric difference [51] between two taxonomic trees counts the number of branches in the first tree which are not present in the second tree plus the number of branches in the second tree which are not present in the first tree. For a tree of $\mathrm{N}$ leaves, the maximal RF distance is $2 \mathrm{~N}-4$ if the trees are rooted and $2 \mathrm{~N}-6$ otherwise.

Figure 3 shows the taxonomic trees corresponding to the three codes implemented in the RODOS system. The RF distances between these trees are as follows (values in brackets represent percentages from the maximum RF distance for $N=24)$ :

$$
\begin{aligned}
A D(p=0.6) & =R F(\text { ATSTEP, DIPCOT } \\
\left.G R S S_{p=0.6}\right) & =16(38 \%) \\
A R(p=0.6) & =R F(\text { ATSTEP, RIMPUFF } \\
\left.G R S S_{p=0.6}\right) & =16(38 \%), \text { and } \\
D R(p=0.6) & =R F(\text { DIPCOT, RIMPUFF } \\
\left.G R S S_{p=0.6}\right) & =4(9.52 \%)
\end{aligned}
$$

This time, the GRSS distance with $p=0.6$ was used to compute the distance matrix. The 0.6 (optimal) value of $p$ was found to give the smallest inter-tree RF distance for the three dispersion codes implemented in the RODOS system. 

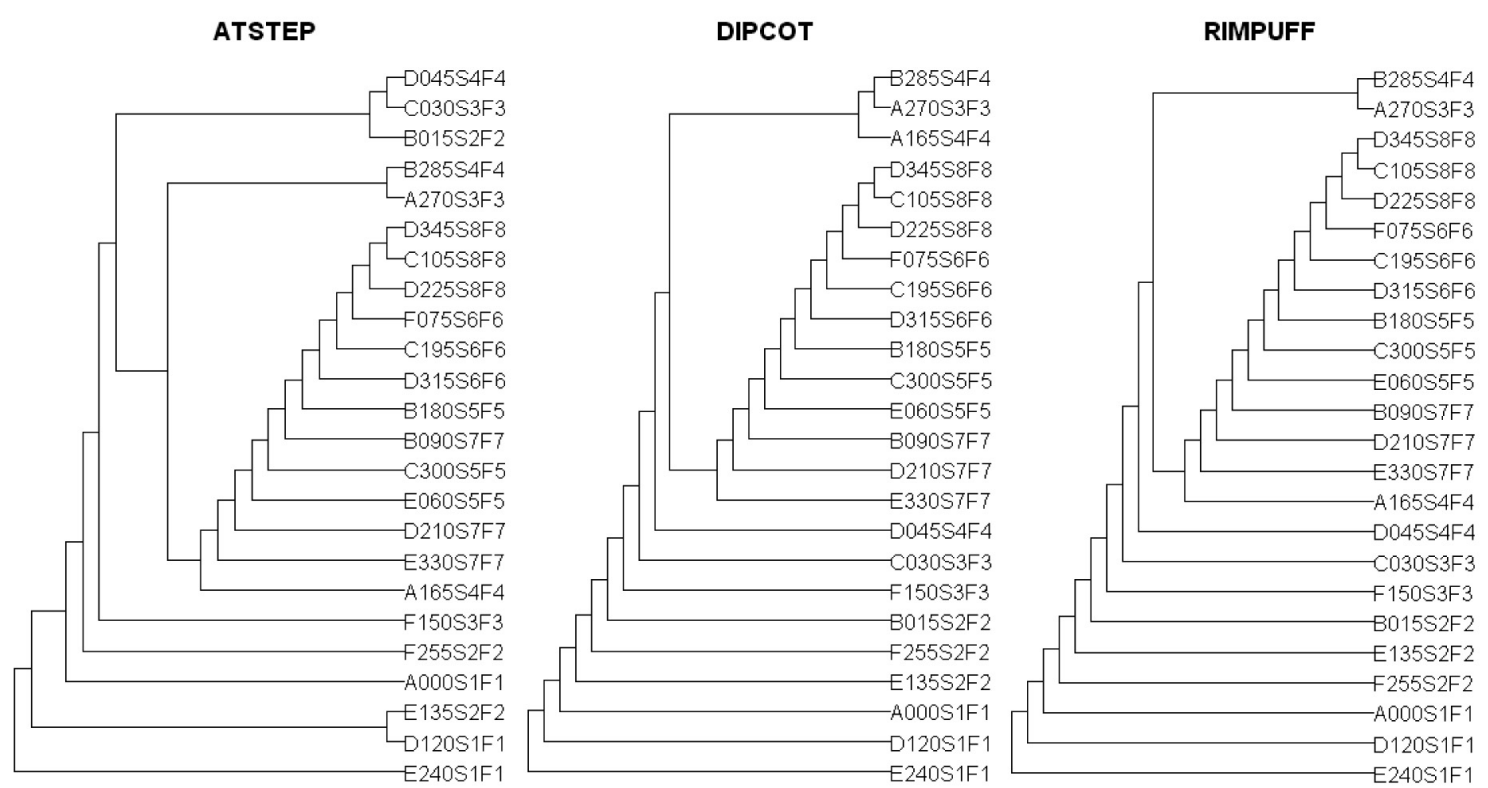

Figure 3. Taxonomic trees corresponding to the three dispersion codes implemented in RODOS. The trees were constructed by means of the GRSS distance with $p=0.6$.

\subsection{New Software Design Diversity Method for the Continuous Verification of Simulation Results}

The workflow supporting the continuous verification of dispersion simulation results depicted in Figure 4 is based on the consensus recovery block algorithm [52], which combines the NVP and Recovery Block methods in one module. The novelty of the proposed method consists in using a database of result taxonomies in order to enable a machine learning process.

The method works as follows: the preprocessing step in the workflow from Figure 4 involves all computations which are usually required for preparing a dispersion simulation (wind and precipitation field computation, source term calculation, etc.) After preprocessing the input case and data, the simulation is performed by two or more functionally redundant dispersion codes. The adjudicator receives the results from all $N \geq 3$ dispersion codes and fetches the reference taxonomies corresponding to each of them from the database; then, it computes the distance between the new result and the ones in the reference ensemble and reconstructs the taxonomic trees starting from the extended distance matrix. The new result is now regarded as a member of the reference ensemble. Next, the adjudicator computes the RF distance between the extended taxonomic trees. The following situations can arise:
- Strict consensus: the RF distances between all taxonomic trees are zero. All results are subjected to an acceptance test. The results passing the acceptance test are forwarded for post-processing;

- Majority consensus: the RF distance is zero within a group containing $(N / 2+1)$ taxonomic trees (called majority consensus group). Only the results in the majority consensus group are subjected to an acceptance test. The results passing the acceptance test are forwarded for post-processing;

- Disagreement: consensus groups only contain $N / 2$ or fewer trees. The user is informed about the disagreement and is asked to make a decision as to which result to take into consideration. This can be done by visual inspection, by analyzing the placement of the new results in the taxonomic trees, or by subjecting all results to the acceptance test.

In this context, a consensus metric can be defined as follows: the number of results (i.e., taxa) present in all the trees divided by the number of results contained in the reference input case ensemble provides an indicator (or metric) for the level of agreement between the participating simulation codes. For the current reference input case ensemble, the consensus agreement level (CAL) between ATSTEP, DIPCOT, 


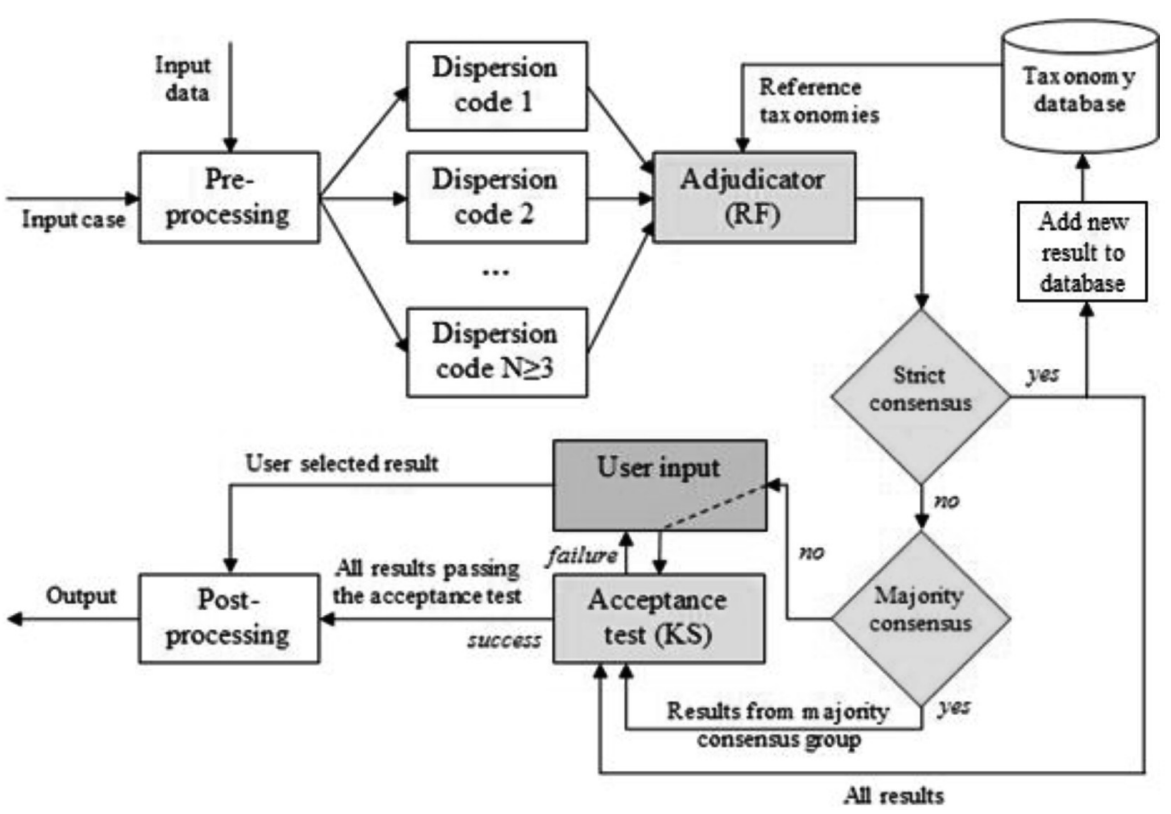

Figure 4. Workflow for a dispersion forecasting system supporting functionally redundant simulation codes and continuous verification of results.

and RIMPUFF is given by CAL (ATSTEP, DIPCOT, RIMPUFF $)=(20 * 100) / 24=83.33 \%$. The sub-tree that is identical for all participating codes will be referred to as the consensus tree (or taxonomy).

Continuous verification is achieved by implementing the tasks depicted in Figure 4 in the dispersion simulation workflow. Whenever a new simulation is carried out using 2 or more simulation codes, the results are added to the result taxonomies of each code stored in the taxonomy database in Figure 4. This way, for each new result the user can be informed whether or not the new result leads to more disagreement among the simulation codes (i.e., by computing the CAL metric). If it does, the user can mark and comment the input case for further investigation by the creators of the simulation codes in question. Thus, the system helps identify input cases, which might reveal software faults upon a more detailed investigation. The results that do not lead to more disagreement between the codes are permanently added to the reference taxonomy database.

\section{Experimental Validation of the Approach}

In order to validate the Kolmogorov-Smirnov acceptance test (KS-AT) and the result-taxono- my-based voter for dispersion simulation results, two versions of the RODOS decision support system have been used. In the following, these two test versions of the system will be referred to as RODOS_v1 and RODOS_v2, respectively. Taking into account that each code implements a different numerical scheme, the RODOS systems is suitable for proving the validity and practical applicability of the conjecture from section 2.6.4 and its implications. Another argument in favor of this choice is the fact that RODOS is being used in several European countries (including Germany) as the official decision support system for nuclear disaster management [53]. The tests using the first version of the RODOS system revealed a number of flaws in the results produced by the three dispersion codes implemented in the RODOS system, notably:

- The gamma dose levels computed by all three RODOS dispersion codes is not invariant with respect to the wind direction even when the topology of the monitored area is leveled;

- For certain input cases, ATSTEP yields zerolevel gamma doses for the first few time steps despite that radioactive materials were released in that time as well;

- DIPCOT computes much smaller doses than ATSTEP and RIMPUFF. 
On the basis of these results the developers of the RODOS system admitted to have identified a fault in the ATSTEP dispersion code and carried out improvement works which concerned DIPCOT as well. These works eventually lead to a new release of the system (i.e., RODOS_v2) which was afterwards made available to the authors. Using the two versions of the systems the exact same set of simulations has been carried out. These simulations were performed using two input case ensembles. The first one is represented by the reference input case ensemble introduced earlier in this work. The second input case ensemble that was used is summarized in Table 2. It contains 24 input cases which represent random combinations of the 24 reference input cases. Two simulated hours are divided in 12 ten-minute time steps. After one simulated hour, the wind direction and speed change while all other parameters stay the same. Using a second input case ensemble with varying wind direction is motivated by the problems that dispersion models typically exhibit in rotating wind situations.

For both versions of the system, the corresponding reference taxonomy was used to verify the input cases from the second input case ensemble described in Table 2. Each input case from this ensemble was regarded as a new simulation result to be verified given the existing operational taxonomy. The procedure employed for accomplishing this task is the one depicted in Figure 4.

Table 2. The second input case ensemble with varying wind speed, direction, and diffusion category.

\begin{tabular}{|c|c|c|c|c|c|c|c|}
\hline \multirow{2}{*}{$\begin{array}{c}\text { Input Case } \\
\text { Id }\end{array}$} & \multicolumn{3}{|c|}{ First simulated hour } & \multicolumn{3}{|c|}{ Second simulated hour } & \\
\cline { 2 - 7 } & $\begin{array}{c}\text { Diffusion } \\
\text { category }\end{array}$ & $\begin{array}{c}\text { Wind direction } \\
\text { [degrees] }\end{array}$ & $\begin{array}{c}\text { Wind speed } \\
\text { [m/s] }\end{array}$ & $\begin{array}{c}\text { Diffusion } \\
\text { category }\end{array}$ & $\begin{array}{c}\text { Wind direction } \\
\text { [degrees] }\end{array}$ & $\begin{array}{c}\text { Wind speed } \\
\text { [m/s }\end{array}$ & $\begin{array}{c}\text { Release } \\
\text { category }\end{array}$ \\
\hline \hline A000B015 & A & 0 & 1 & B & 15 & 2 & F1 \\
A165B090 & A & 165 & 4 & B & 165 & 7 & F4 \\
A270B180 & A & 270 & 3 & B & 270 & 5 & F3 \\
B015B285 & B & 15 & 2 & B & 285 & 4 & F2 \\
B090C030 & B & 90 & 7 & C & 30 & 3 & F7 \\
B180C105 & B & 180 & 5 & C & 105 & 8 & F5 \\
B285C195 & B & 285 & 4 & C & 195 & 6 & F4 \\
C030C300 & C & 30 & 3 & C & 300 & 5 & F3 \\
C105D045 & C & 105 & 8 & D & 45 & 4 & F8 \\
C195D120 & C & 195 & 6 & D & 120 & 1 & F6 \\
C300D210 & C & 300 & 5 & D & 210 & 7 & F5 \\
D045D225 & D & 45 & 4 & D & 225 & 8 & F4 \\
D120D315 & D & 120 & 1 & D & 315 & 6 & F1 \\
D210D345 & D & 210 & 7 & D & 345 & 8 & F7 \\
D225E060 & D & 225 & 8 & E & 60 & 5 & F8 \\
D315E135 & D & 315 & 6 & E & 135 & 2 & F6 \\
D345E240 & D & 345 & 8 & E & 240 & 1 & F8 \\
E060E330 & E & 60 & 5 & E & 330 & 7 & F5 \\
E135F075 & E & 135 & 2 & F & 75 & 6 & F2 \\
E240F150 & E & 240 & 1 & F & 150 & 3 & F1 \\
E330F255 & E & 330 & 7 & F & 255 & 2 & F7 \\
F075A000 & F & 75 & 6 & A & 0 & 1 & F6 \\
F150A165 & F & 150 & 3 & A & 165 & 4 & F3 \\
F255A270 & F & 255 & 2 & A & 270 & 3 & F2 \\
\hline & & & & & & & \\
\hline
\end{tabular}




\subsection{Results}

For each version of the RODOS system the KS test was first run on the basis of the two input case ensembles. The KS test can be applied at a significance level (alpha) 0.01, 0.05, $0.1,0.15$, etc. In practical terms, using alpha $=0.01$ provides a less strict assessment than using alpha $=0.05$. Tests using alpha $=0.05$ lead to the rejection of more input cases than for alpha $=0.01$ and it would have been more difficult to find correlations between the output of the voter and that of the acceptance test. However, we do recommend using a significance level of 0.05 if a more scrutinizing assessment of the trustworthiness of simulation results is aimed at, especially when the number of passing cases is getting closer to $100 \%$.

The results of these tests are summarized in Table 3 (figures in boldface indicate an improvement from one version of the simulation code to another). They show that the KS test is sensitive to the improvements brought to the RODOS_v2 system. The only exception is produced by the DIPCOT simulation code for the reference input case ensemble. In this case, the developers might have introduced a new fault while attempting to correct others. Notably, the net improvement of ATSTEP_v2 over ATSTEP_v1 reflects the efforts of the developers to remove the flaws revealed by our previous verification analysis of the system.

Next, the consensus taxonomies for the two versions of the systems were inferred on the basis on the reference input case ensemble. This is a prerequisite for performing the tests on the input case ensemble with rotating wind direction. This yielded a 20-leaf consensus tree for the RODOS_v1 simulation codes and a 21-leaf consensus tree for the RODOS_v2 simulation codes. In other words, the consensus agreement level for the two versions of the system was $C A L$ (ATSTEP_v1,DIPCOT_v1,RIMPUFF_v1) = $83.33 \%$ and CAL (ATSTEP_v2,DIPCOT_v2, RIMPUFF_v2) $=87.5 \%$, respectively.

These high consensus agreement levels themselves support the argument that the conjecture from section 2.6.4 holds for the dispersion codes implemented in both versions of the RODOS system. Moreover, the increase in the consensus agreement level between the codes from the second version of the system provides evidence that the CAL metric is sensitive to the removal of software faults from the simulation codes under investigation. Table 4 summarizes the consensus recovery block's output for the results of the two versions of the RODOS system for the 24 input cases from the test ensemble with varying weather conditions. In order to ensure equity for the comparison, an 18-taxon consensus tree (i.e., a sub-taxonomy of the consensus trees corresponding to the two versions of the system) correlated with the KolmogorovSmirnov test results was used with both versions of the RODOS system. The taxonomy based voter output clearly reveals an improvement of RODOS_v2 over RODOS_v1. While the total RF distance for the pair ATSTEP-DIPCOT (AD) remains constant from one version to another, the pairs ATSTEP-RIMPUFF (AR) and DIPCOT-RIMPUFF (DR) yield smaller total RF distances (i.e., summed up over all input cases). The improvement for the pair DR is quite significant. While the improvements brought to the ATSTEP code were clearly reflected by the KS test results, the smaller total RF distance for the pair DIPCOT-RIMPUFF reflects the improvements in DIPCOT_v2 over DIPCOT_v1.

Table 3. Kolmogorov-Smirnov test results for the two input case ensembles for RODOS_v1 and RODOS_v2.

\begin{tabular}{|c|c|c|c|c|c|c|}
\hline \multicolumn{7}{|c|}{ Reference Input Case Ensemble } \\
\hline \hline$\alpha=1 \%$ & ATSTEP_v1 & ATSTEP_v2 & DIPCOT_v1 & DIPCOT_v2 & RIMPUFF_v1 & RIMPUFF_v2 \\
\hline Passed & $16 / 24$ & $22 / 24$ & $20 / 24$ & $19 / 24$ & $21 / 24$ & $21 / 24$ \\
\hline Percent & $66.67 \%$ & $\mathbf{9 1 . 6 7 \%}$ & $83.33 \%$ & $79.17 \%$ & $87.50 \%$ & $87.50 \%$ \\
\hline \hline \multicolumn{7}{|c|}{ Second Input Case Ensemble } \\
\hline \hline$\alpha=1 \%$ & ATSTEP_v1 & ATSTEP_v2 & DIPCOT_v1 & DIPCOT_v2 & RIMPUFF_v1 & RIMPUFF_v2 \\
\hline Passed & $17 / 24$ & $20 / 24$ & $15 / 24$ & $19 / 24$ & $20 / 24$ & $23 / 24$ \\
\hline Percent & $70.83 \%$ & $\mathbf{8 3 . 3 3 \%}$ & $62.50 \%$ & $\mathbf{7 9 . 1 7 \%}$ & $83.33 \%$ & $\mathbf{9 5 . 8 3 \%}$ \\
\hline
\end{tabular}


Table 4. The strict consensus recovery block output for the 24 input cases with varying weather conditions for RODOS_v1 and RODOS_v2. Abbreviations: AD = RF(ATSTEP, DIPCOT); AR = RF(ATSTEP, RIMPUFF); $\mathrm{DR}=\mathrm{RF}(\mathrm{DIPCOT}, \mathrm{RIMPUFF}) ; \mathrm{RF}=$ Robinson-Foulds distance; VOTE $=$ output of the taxonomy based voter for some given input case; AT = results of the Kolmogorov-Smirnov acceptance test; AT\&VOTE = intersection of AT and VOTE output sets; A = ATSTEP; D = DIPCOT; $\mathrm{R}=$ RIMPUFF; Zeroes = number of input cases for which the RF distance between the respective two simulation codes is zero.

\begin{tabular}{|c|c|c|c|c|c|c|c|c|c|c|c|c|}
\hline \multirow[b]{2}{*}{ Input case } & \multicolumn{6}{|c|}{ RODOS_v1 } & \multicolumn{6}{|c|}{ RODOS_v2 } \\
\hline & AD & $\mathbf{A R}$ & DR & VOTE & AT & AT \& VOTE & AD & AR & DR & VOTE & AT & AT \& VOTE \\
\hline A000B015 & 0 & 0 & 0 & ADR & $\mathrm{R}$ & $\mathrm{R}$ & 0 & 0 & 0 & ADR & ADR & ADR \\
\hline A165B090 & 0 & 2 & 2 & $\mathrm{AD}$ & AR & A & 0 & 0 & 0 & ADR & DR & DR \\
\hline A270B180 & 2 & 2 & 4 & - & AR & - & 0 & 0 & 0 & ADR & $\mathrm{ADR}$ & ADR \\
\hline В015B285 & 0 & 2 & 2 & $\mathrm{AD}$ & ADR & $\mathrm{AD}$ & 2 & 2 & 0 & $\mathrm{DR}$ & ADR & $\mathrm{DR}$ \\
\hline В090С030 & 0 & 2 & 2 & $\mathrm{AD}$ & ADR & $\mathrm{AD}$ & 0 & 0 & 0 & ADR & ADR & ADR \\
\hline B180C105 & 2 & 0 & 2 & $\mathrm{AR}$ & ADR & AR & 2 & 2 & 0 & DR & ADR & DR \\
\hline B285C195 & 4 & 2 & 6 & - & ADR & - & 0 & 2 & 2 & $\mathrm{AD}$ & ADR & $\mathrm{AD}$ \\
\hline C030C300 & 0 & 0 & 0 & ADR & ADR & ADR & 0 & 0 & 0 & ADR & $\mathrm{ADR}$ & ADR \\
\hline C105D045 & 0 & 0 & 0 & ADR & $\mathrm{ADR}$ & $\mathrm{ADR}$ & 0 & 0 & 0 & ADR & ADR & ADR \\
\hline C195D120 & 0 & 0 & 0 & ADR & $\mathrm{R}$ & $\mathrm{R}$ & 2 & 0 & 2 & $\mathrm{AR}$ & DR & $\mathrm{R}$ \\
\hline C300D210 & 0 & 0 & 0 & ADR & ADR & ADR & 0 & 0 & 0 & ADR & ADR & $\mathrm{ADR}$ \\
\hline D045D225 & 0 & 0 & 0 & ADR & DR & $\mathrm{DR}$ & 0 & 0 & 0 & ADR & $\mathrm{AR}$ & $\mathrm{AR}$ \\
\hline D120D315 & 0 & 0 & 0 & ADR & - & - & 0 & 0 & 0 & ADR & ADR & ADR \\
\hline D210D345 & 0 & 0 & 0 & ADR & DR & DR & 0 & 0 & 0 & ADR & $\mathrm{AR}$ & $\mathrm{AR}$ \\
\hline D225E060 & 2 & 2 & 0 & $\mathrm{DR}$ & ADR & DR & 0 & 2 & 2 & $\mathrm{AD}$ & ADR & $\mathrm{AD}$ \\
\hline D315E135 & 0 & 2 & 2 & $\mathrm{AD}$ & $\mathrm{R}$ & - & 2 & 2 & 0 & DR & $\mathrm{R}$ & $\mathrm{R}$ \\
\hline D345E240 & 0 & 2 & 2 & $\mathrm{AD}$ & $\mathrm{AR}$ & A & 0 & 2 & 2 & $\mathrm{AD}$ & ADR & $\mathrm{AD}$ \\
\hline E060E330 & 0 & 0 & 0 & ADR & ADR & ADR & 0 & 0 & 0 & ADR & ADR & $\mathrm{ADR}$ \\
\hline E135F075 & 0 & 0 & 0 & ADR & $\mathrm{AD}$ & $\mathrm{AD}$ & 2 & 2 & 0 & DR & ADR & DR \\
\hline E240F150 & 0 & 0 & 0 & ADR & $\mathrm{AD}$ & $\mathrm{AD}$ & 2 & 0 & 2 & $\mathrm{AR}$ & $\mathrm{A}$ & A \\
\hline E330F255 & 0 & 2 & 2 & $\mathrm{AD}$ & $\mathrm{AD}$ & $\mathrm{AD}$ & 0 & 0 & 0 & ADR & A & A \\
\hline F075A000 & 2 & 0 & 2 & $\mathrm{AR}$ & DR & $\mathrm{R}$ & 0 & 2 & 2 & $\mathrm{AD}$ & $\mathrm{DR}$ & $\mathrm{D}$ \\
\hline F150A165 & 0 & 0 & 0 & ADR & $\mathrm{AR}$ & DR & 0 & 0 & 0 & ADR & $\mathrm{ADR}$ & $\mathrm{ADR}$ \\
\hline F255A270 & 0 & 0 & 0 & ADR & $\mathrm{AR}$ & $\mathrm{AR}$ & 0 & 0 & 0 & ADR & ADR & ADR \\
\hline Total & 12 & 18 & 26 & & & & 12 & 16 & 12 & & & \\
\hline Zeroes & 19 & 15 & 14 & & & & 18 & 16 & 18 & & & \\
\hline
\end{tabular}

Figure 5 shows the exact percentages of the cases when each of the three possible outcomes (i.e., strict consensus / majority consensus / disagreement) is produced by the taxonomybased voter for RODOS_v1 and RODOS_v2. Thanks to removing some of ATSTEP's and DIPCOT's software faults, the results for RODOS_v2 no longer yield any cases of disagreement. The $8 \%$ disagreement share is split up in two fractions; one of them is gained by the strict consensus share, and the other one by the majority consensus share. There also appears to be no improvement/deterioration pattern for the voter's output except for the absence of disagreement cases from RODOS_v2's results.

Figure 6 shows the percentage ratios of the 4 possible output types of the consensus recovery block (i.e., AT\&VOTE column in ) for both versions of the system:

- Consensus (labeled "ADR" in Table 4): the results from all three codes are forwarded for post-processing; 

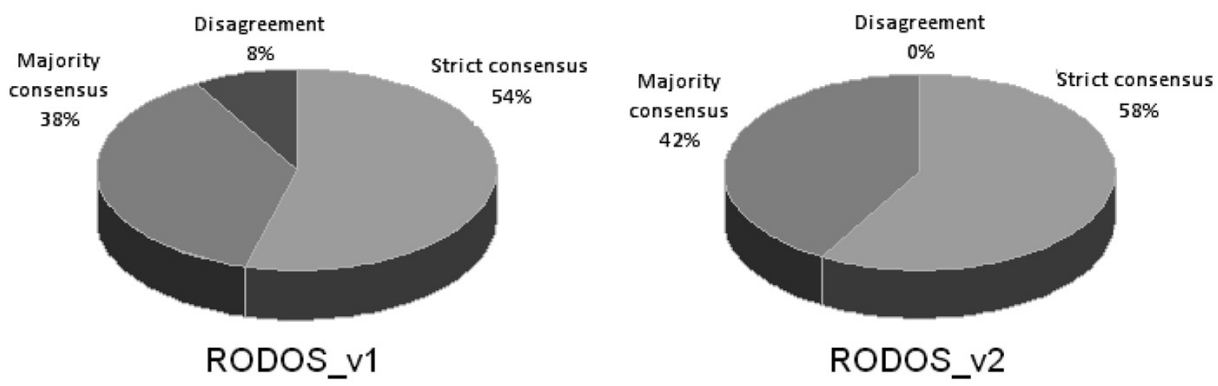

Figure 5. Strict consensus / majority consensus / disagreement percentage ratios resulting from the taxonomy-based voter for the two RODOS versions for the 24 input cases with varying weather conditions.
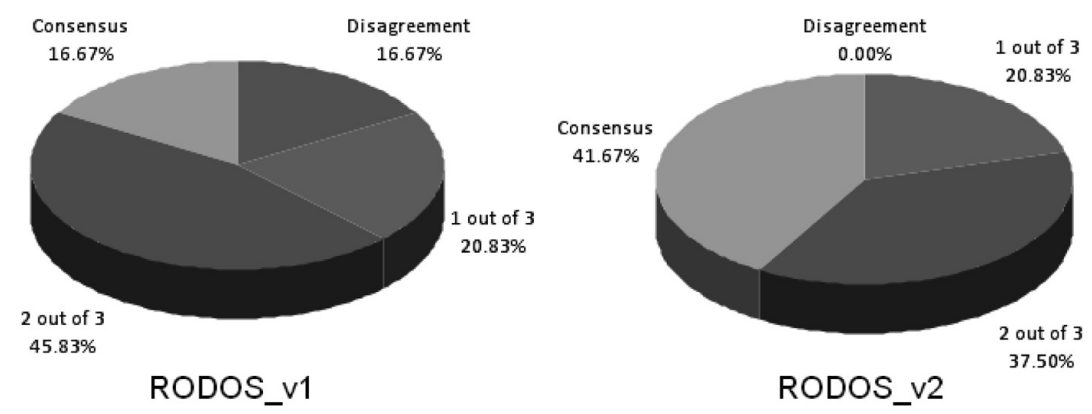

Figure 6. Consensus / 2 out of 3 / 1 out of 3 / disagreement percentage ratios resulting from the output of the consensus recovery block (i.e., AT\&VOTE column in Table 4) for the two RODOS versions for the 24 input cases with varying weather conditions.

- 2 out of 3 (labels "AD" for the pair ATSTEPDIPCOT, "AR" for ATSTEP-RIMPUFF, and "DR" for DIPCOT-RIMPUFF): the results from two out of three codes are forwarded for post-processing;

- 1 out of 3 (labeled "A" for ATSTEP, "D" for DIPCOT, and " $R$ " for RIMPUFF): the result from one out of three codes is forwarded for post-processing;

- Disagreement (labeled “-”): the result from none of the codes is automatically forwarded for post-processing. The user is asked to make a decision.

Once again, for RODOS_v2 the consensus recovery block's output does not yield any cases of disagreement. For RODOS_v2, in $79.17 \%$ of the cases the user gets a consensual or " 2 out of 3" recommendation from the system. This positive evolution for RODOS_v2 is due to a better correlation of the voter's output with the acceptance test results compared to RODOS_v1's case.

\section{Conclusion}

In this paper we argued that DSNE systems are safety-critical and should thus be treated as such throughout their lifecycle. For this reason, means for the continuous verification should be in place for as long as the system is being developed, maintained, and used. To this end, we introduced a new software design diversity method for continuous verification, which adds a machine learning capability to a consensus recovery block method from the field of software reliability engineering. To enable the machine learning capability, we developed a new acceptance test and a new taxonomy-based voting scheme for dispersion simulation results. The Consensus Recovery Block method was also modified to support the machine learning capability of the voting system using a database of result taxonomy. The experimental validation of these methods was based on the widely used RODOS DSNE system. By using two consecutive versions of the RODOS systems we showed that the acceptance test and the taxonomy based voter clearly reflect the improvements brought to the RODOS simulation codes from one version to another, thus being able to signal the 
existence of latent software faults in simulation codes. The proposed approach thus helps finding further latent software faults in existing DSNE systems, such as the RODOS and the ENSEMBLE systems.

Considering that real data is the only gold standard in the evaluation of atmospheric dispersion simulation software, we intend to repeat the experiment using the post-Fukushima version of the RODOS and possibly other systems based on a reference input case ensemble, which includes the Fukushima data sets. For example, input case ensembles can be generated by varying different parameters of the Fukushima data set. Such a study builds upon the approaches used in [31] and [30], while providing additional means for the application of verification criteria (such as the Gaussian boundary condition, the mass conservation principle, etc.) using the methods introduced in this work.

\section{References}

[1] S. Galmarini et al., "Ensemble dispersion forecasting, Part I: concept, approach and indicators", Atmospheric Environment 38, pp. 4607-4617, 2004. http://dx.doi.org/10.1016/j.atmosenv.2004.05.031

[2] N. A. a. S. A. NASA, "NASA Standard for Software Assurance (NASA-STD-8739.8)", NASA, Washington, D.C., 2004.

[3] P. E. Farrell et al., "Automated continuous verification for numerical simulation", Geoscientific Model Development, vol. 4, pp. 435-449, 2011. http://dx.doi.org/10.5194/gmd-4-435-2011

[4] A. Avizienis and J. C. Laprie, "Dependable computing: From concepts to design diversity", Proceedings of the IEEE 74, 1986, pp. 629-638. http://dx.doi.org/10.1109/PROC.1986.13527

[5] T. Anderson and R. Kerr, "Recovery blocks in action: A system supporting high reliability", in Proceedings of the 2nd international conference on Software engineering, Los Alamitos, 1976.

[6] J. Ehrhardt and A. Weis, "RODOS: Decision Support System for Off-site Nuclear Emergency Management in Europe", Directorate-General for Research, European Commission, Luxemburg, 2000.

[7] D. Etling, "Theoretische Meteorologie", Eine Einfuhrung, Berlin-Heidelberg: Springer-Verlag, 2008.

[8] D. Etling et al., "Application of a random walk model to turbulent diffusion in complex terrain", Atmospheric Environment, vol. 20, pp. 741-747, 1986. http://dx.doi.org/10.1016/00046981(86)90188-5
[9] Verein Deutscher Ingenieure, "Environmental meteorology, Atmospheric dispersion models (Particle model - VDI 3945)", VDI, Düsseldorf, 2000.

[10] S. Andronopoulos and E. Davakis, "RODOSDIPCOT Model Description and Evaluation", KIT, Karlsruhe, 2009.

[11] S. Thykier-Nielsen et al., "Description of the Atmospheric Dispersion Module RIMPUFF", KIT, Karlsruhe, 1999.

[12] J. Päsler-Sauer, "Description of the Atmospheric Dispersion Model ATSTEP”, KIT, Karlsruhe, 2000.

[13] W. L. Oberkampf and T. G. Trucano, "Verification and validation in computational fluid dynamics", Progress in Aerospace Sciences, vol. 38, 2002, pp. 209-272. http://dx.doi.org/10.1016/S03760421(02)00005-2

[14] L. Hatton and A. Roberts, "How accurate is scientific software?", IEEE Transactions on Software Engineering, vol. 20, pp. 785-797, 1994. http://dx.doi.org/10.1109/32.328993

[15] D. P. Holzworth et al., "Simple software processes and tests improve the reliability and usefulness of a model", Environmental Modelling \& Software, vol. 26, pp. 510-516, 2011.

http://dx.doi.org/10.1016/j.envsoft.2010.10.014

[16] A. J. Jakeman et al., "Ten iterative steps in development and evaluation of environmental models", Environmental Modelling \& Software, vol. 21, pp. 602-614, 2006.

http://dx.doi.org/10.1016/j.envsoft.2006.01.004

[17] D. Kelly et al., "Examining random and designed tests to detect code mistakes in scientific software", Journal of Computational Science, vol. 2, pp. 47-56, 2011.

http://dx.doi.org/10.1016/j.jocs.2010.12.002

[18] Z. Merali, "Computational science: Error, why scientific programming does not compute", Nature 467, pp. 775-777, 2010. http://dx.doi.org/10.1038/467775a

[19] D. Post and L. Votta, "Computational Science Demands a New Paradigm", Physics Today, vol. 58, pp. 35-41, 2005.

http:/ / dx.doi.org/10.1063/1.1881898

[20] P. Godefroid et al., "DART: directed automated random testing", ACM Sigplan Notices, vol. 40, pp. 213-223, 2005.

http://dx.doi.org/10.1145/1064978.1065036

[21] M. Grindal et al., "Combination testing strategies: a survey", Software Testing, Verification and Reliability, vol. 15, no. 3, pp. 167-199, 2005. http://dx.doi.org/10.1002/stvr.319

[22] D. Hook and D. Kelly, "Testing for trustworthiness in scientific software", in ICSE Workshop on Software Engineering for Computational Science and Engineering, Vancouver, BC, 2009. http://dx.doi.org/10.1109/SECSE.2009.5069163 
[23] E. Davakis et al., "Validation of DIPCOT-II model based on the Kincaid experiment", International Journal of Environment and Pollution, vol. 14, pp. 131-142, 2000.

http://dx.doi.org/10.1504/IJEP.2000.000534

[24] E. Davakis et al., "Validation study of the dispersion Lagrangian particle model DIPCOT over complex topographies using different concentration calculation methods", International journal of environment and pollution, vol. 20, pp. 33-46, 2003. http://dx.doi.org/10.1504/IJEP.2003.004242

[25] D. Arnold et al., "Comparison of the dispersion model in rodos-lx and mm5-v3. 7-flexpart (v6. 2). A case study for the Nuclear Power Plant of Almaraz", Hrvatski meteorološki časopis 43, pp. 485-490, 2008.

[26] J. Brandt et al., "Numerical modelling of transport, dispersion, and depositionñvalidation against ETEX-1, ETEX-2 and Chernobyl", Environmental Modelling \& Software, vol. 15, pp. 521-531, 2000. http://dx.doi.org/10.1016/S13648152(00)00035-9

[27] J. Päsler-Sauer, "Comparison and validation exercises of the three atmospheric dispersion models in RODOS", Radioprotection, vol. 45, pp. S89-S96, 2010.

http://dx.doi.org/10.1051/radiopro/2010018

[28] I. V. Kovalets et al., "alculation of the far range atmospheric transport of radionuclides after the Fukushima accident with the atmospheric dispersion model MATCH of the JRODOS system", International Journal of Environment and Pollution, vol. 15, pp. 101-109, 2014.

http:/ /dx.doi.org/10.1504/IJEP.2014.065110

[29] Á. Leelôssy et al., "Short and long term dispersion patterns of radionuclides in the atmosphere around the Fukushima Nuclear Power Plant," Journal of environmental radioactivity, vol. 102, pp. 1117-1121, 2011.

http://dx.doi.org/10.1016/j.jenvrad.2011.07.010

[30] F. Gering et al., "Potential consequences of the Fukushima accident for off-site nuclear emergency management: a case study for Germany.," Radiat Prot Dosimetry, vol. 155, pp. 146-154, 2013. http://dx.doi.org/10.1093/rpd/ncs323

[31] C. Von Arx et al., "Comparison of operational atmospheric dispersion models in Germany," in 16th International Conference on Harmonisation within Atmospheric Dispersion Modelling for Regulatory Purposes, Varna, 2014.

[32] A. Riccio et al., "Seeking for the rational basis of the median model: the optimal combination of multi-model ensemble results," Atmospheric Chemistry and Physics, vol. 7, pp. 6085-6098, 2007. http://dx.doi.org/10.5194/acp-7-6085-2007

[33] E. Solazzo et al., "Pauci ex tanto numero: reduce redundancy in multi-model ensembles," Armospheric Chemistry and Physics, vol. 13, pp. 8315-8333, 2013.

http://dx.doi.org/10.5194/acp-13-8315-2013
[34] I. Kioutsioukis and S. Galmarin, "De praeceptis ferendis: good practice in multi-model ensembles," Atmos. Chem. Phys., vol. 14, pp. 11791-11815, 2014. http://dx.doi.org/10.5194/acp-14-11791-2014

[35] S. Potempski and S. Galmarini, "Est modus in rebus: analytical properties of multi-model ensembles," Atmos. Chem. Phys., vol. 9, pp. 9471-9489, 2009. http://dx.doi.org/10.5194/acp-9-9471-2009

[36] J. C. Leveson and N. G. Knight, "A Large Scale Experiment In N-Version Programming," in Digest of Papers FTCS-15: Fifteenth International Symposium on Fault-Tolerant Computing, Ann Arbor, MI, 1985.

[37] J. C. Knight and N. G. Leveson, "An Experimental Evaluation of the Assumption of Independence in Multi-version Programming," IEEE Transactions on Software Engineering, vol. 12, pp. 96-109, 1986. http://dx.doi.org/10.1109/TSE.1986.6312924

[38] M. R. Lyu, Handbook of Software Reliability Engineering. Maidenh: McGraw-Hill, 1996.

[39] D. E. Eckhardt and L. D. Lee, "A theoretical basis for the analysis of multiversion software subject to coincident errors," IEEE Transactions on Software Engineering, vol. 12, pp. 1511-1517, 1985. http://dx.doi.org/10.1109/TSE.1985.231895

[40] D. T. P. Brière, “AIRBUS A320/A330/A340 electrical flight controls-A family of fault-tolerant systems," in Fault-Tolerant Computing FTCS-23. Digest of Papers, IEEE, 1993. http:/ /dx.doi.org/10.1109/ftcs.1993.627364

[41] D. F. McAllister and M. A. Vouk, Handbook of Software Reliability Engineering. Maidenh: McGrawHill, 1996.

[42] B. Littlewood et al., "Modeling software design diversity: a review," ACM Computing Surveys, vol. 33, 2001, pp. 177-208.

http://dx.doi.org/10.1145/384192.384195

[43] A. Avizienis, "The N-Version Approach to FaultTolerant Software," IEEE Trans. Softw. Eng., vol. 11, pp. 1491-1501, 1985. http://dx.doi.org/10.1109/TSE.1985.231893

[44] H. Hecht, "Fault-Tolerant Software for Real-Time Applications," ACM Comput. Surv., vol. 8, pp. 391407, 1976.

http://dx.doi.org/10.1145/356678.356681

[45] W. Weibull, "A statistical distribution function of wide applicability," J. Appl. Mech.-Trans. ASME, vol. 18, pp. 293-297, 1951.

[46] K. Apt, "Applicability of the Weibull distribution function to atmospheric radioactivity data," Atmospheric Environment, vol. 10, pp. 777-781, 1967. http://dx.doi.org/10.1016/00046981(76)90079-2

[47] F. J. Massey, "The Kolmogorov-Smirnov Test for Goodness of Fit," Journal of the American Statistical Association, vol. 46, pp. 68-78, 1951. http://dx .doi.org/10.1080/01621459.1951.10500769 
[48] J. McDonald, Handbook of biological statistics, Baltimore. MD: Sparky House Publishing, 2009.

[49] J. A. Hartigan, Clustering algorithms. New York: John Wiley \& Sons, 1975.

[50] Bundesministerium für Umwelt, Naturschutz und Reaktorsicherheit, "Leitfaden für den Fachberater Strahlenschutz der Katastrophenschutzleitung bei kerntechnischen Notfällen", München-Jena: Elsevier Urban \& Fischer, 2004.

[51] D. R. Robinson and L. R. Foulds, "Comparison of phylogenetic trees," Mathematical Biosciences, vol. 53, pp. 131-147, 1981. http://dx.doi.org/10.1016/ 0025-5564(81)90043-2

[52] M. A. Vouk et al., "An Empirical Evaluation of Consensus Voting and Consensus Recovery Block Reliability in the Presence of Failure Correlation,' Journal of Computer and Software Engineering, vol. 1, pp. 367-388, 1993.

[53] C. R. Palma, “Off-site Nuclear Emergency Management and Restoration of Contaminated Environments", Brussels: European Commission, 2007.

Received: May, 2015

Revised: July, 2015

Accepted: July, 2015
Contact addresses:

Tudor B. Ionescu Institut für Kernenergetik und Energiesysteme (IKE)

Pramergasse 26/14

1090-Vienna

Austria

e-mail: tudor.b.ionescu@gmail.com

Walter Scheuermann Institut für Kernenergetik und Energiesysteme (IKE)

Pfaffenwaldring 31 70569-Stuttgart Germany

e-mail: scheuermann@ike.uni-stuttgart.de

TUDOR B. IONESCU is a software engineer with industry experience in safety-critical software development. He received his doctorate degree from the University of Stuttgart. He is now employed as a senior software architect at SIEMENS. His research interests include pattern-oriented software architecture, non-functional requirements, fault-tolerant computing, flexible workflow systems, web-based technologies, and distributed computing.

WALTER SCHEUERMANN has received his doctorate degree from the University of Stuttgart and is now the head of the Knowledge Engineering Department of the Institute of Nuclear Technology and Energy Systems of the University of Stuttgart, Germany. His research interests are simulation of complex systems, dispersion modeling and simulation, and parallel and distributed computing. 
